\title{
Kawasaki Shock Syndrome and Covid-19
}

\author{
Sana Ashraf, Farah Shams Abbasi and Mehnaz Atiq \\ Department of Pediatrics, Liaquat National Hospital, Karachi, Pakistan
}

\begin{abstract}
Kawasaki disease (KD) is a systemic vasculitis of unknown cause affecting children under 5 years of age. It is thought to be triggered by several viruses. Recently, Kawasaki-like disease has been reported worldwide in patients with COVID-19, giving rise to a new term of multi-system inflammatory syndrome in children (MIS-C). We report a case of a previously healthy 7-year boy, referred to our hospital with provisional diagnosis of measles due to generalised erythematous, maculopapular rash and conjunctivitis. On detailed evaluation, the patient fulfilled the clinical and laboratory criteria of MIS-C, and COVID-19 antibodies were positive. He was treated successfully with high dose of intravenous immunoglobulins (IVIG) and methylprednisolone. Patient was followed one week later with repeat echocardiography, which showed improvement. In conclusion, early recognition and timely treatment can prevent adverse outcomes in MIS-C.
\end{abstract}

Key Words: COVID-19, Kawasaki disease, MIS-C.

How to cite this article: Ashraf S, Abbasi FS, Atiq M. Kawasaki Shock Syndrome and Covid-19. J Coll Physicians Surg Pak 2021; 31(JCPSPCR):CR135-CR137.

\section{INTRODUCTION}

Kawasaki disease (KD) is a systemic vasculitis of unknown cause, primarily affecting the pediatric population younger than 5 years of age. It was first described by Tomisaku Kawasaki in 1967 in Japan. The etiology is still unknown; but the disease is triggered by several viruses. ${ }^{1}$

The cardiac complications of KD are well known. There may be atypical clinical presentations of KD, including shock. During COVID-19 pandemic, several recent clinical reports have appeared in literature on Kawasaki-like disease in children due to COVID-19. The multi-system inflammatory syndrome in children (MIS-C) noted in COVID-19 has been associated with KD. Such cases have been reported both nationally and internationally. ${ }^{1-4}$

We, herein, report a case of MIS-C in a 7-year boy who was initially referred as a measlescase; but correctly diagnosed and successfully treated at our centre.

\section{CASE REPORT}

A previously healthy 7-year boy was referred to our hospital with a provisional diagnosis of measles, as he had generalised erythematous maculopapular rash and conjunctivitis. He had high grade fever, documented up to $101^{\circ} \mathrm{F}$ associated with chills and rigors, headache, neck pain for one week, rashes all the over the body from the 4th day of illness plus eye swelling and redness.

Correspondence to: Dr. Sana Ashraf, Department of Pediatrics, Liaquat National Hospital, Karachi, Pakistan

E-mail:dr.sana016@gmail.com

Received: December 24, 2020; Revised: May 18, 2021;

Accepted: June 04, 2021

DOI: https://doi.org/10.29271/jcpsp.2021.JCPSPCR.CR135
On physical examination, he was irritable, with non-pruritic maculopapular rash over torso and upper limbs, non-exudative conjunctivitis along with edema of hands and feet and cracked lips. There was no hepatosplenomegaly or lymphadenopathy. On arrival, he was febrile, hypotensive, tachypneic, tachycardiac with weak peripheral pulses and poor perfusion. He was resuscitated in the Emergency Room and admitted in the Pediatric Intensive Care unit with a provisional diagnosis of Kawasaki-shock syndrome, based on symptoms and physical findings.

Initial laboratory investigations showed mild anemia (Hemoglobin, $9.3 \mathrm{~g} / \mathrm{dl})$, increased total leucocyte count $\left(30 \times 10^{9} / \mathrm{L}\right)$ with predominant neutrophils (86\%), Iymphopenia (6\%) and low normal platelets of $102 \times 10^{9} / \mathrm{L}$. C-reactive protein (CRP) was $24 \mathrm{mg} / \mathrm{dl}$, albumin $1.76 \mathrm{~g} / \mathrm{dl}$, serum sodium $126 \mathrm{mEq} / \mathrm{L}$, urea 99 $\mathrm{mg} / \mathrm{dl}$ and creatinine was $1.6 \mathrm{mg} / \mathrm{dl}$. Other relevant work-up included raised serum ferritin level of $7,909 \mathrm{ng} / \mathrm{ml}$, raised lactate dehydrogenase (LDH) $385 \mathrm{U} / \mathrm{L}$, raised serum cardiac markers pro-BNP, 10,879 pg/ml and troponin I, $1 \mathrm{ng} / \mathrm{ml}, \mathrm{D}$ dimers $11.56 \mathrm{mg} / \mathrm{L}$ and mild ly elevated liver enzymes(Table I).

Echocardiography was done, which showed left ventricular enlargement with mild systolic dysfunction, ejection fraction of $56 \%$, mildly dilated right coronary artery (Z score +2.9$)$, high normal size of left main coronary artery $(Z$ score +2.3$)$, with hyper-echoic coronary endothelium and normal tapering. There was mild circumferential pericardial effusion measuring $9 \mathrm{~mm}$ with a trace to mild mitral regurgitation (Figure 1 ).

There was no history of recent travel or contact with COVID-19, but as the child fulfilled the clinical criteria of KD and MIS-C, COVID-19 infection was suspected and a COVID PCR and antibodies were sent. PCR was negative but antibodies were positive. 
Table I: Serial laboratory workup.

\begin{tabular}{|c|c|c|c|}
\hline & Day 1 & Day 3 & Day 5 \\
\hline Hemoglobin (g/dl) /packed cell volume (\%) & $9.3 / 28$ & $7.8 / 24$ & \\
\hline Total leukocyte count $\left(10^{9} / \mathrm{L}\right)(\mathrm{N} \%, \mathrm{~L} \%)$ & $30(85,6.8)$ & $18.6(86,6)$ & $13.6(74,22)$ \\
\hline Platelets $\left(10^{9} / \mathrm{L}\right)$ & 102 & 139 & 167 \\
\hline Urea (mg/dl) & 99 & 115 & 117 \\
\hline Creatinine (mg/dl) & 1.6 & 1.23 & 0.90 \\
\hline Sodium (m.mol/L) & 124 & 129 & 136 \\
\hline CRP $(\mathrm{mg} / \mathrm{dl})$ & 24 & & 8.68 \\
\hline Albumin (g/dl) & 2.23 & 1.76 & 3.03 \\
\hline NT PRO BNP (pg/ml) & 9533 & & 10879 \\
\hline $\operatorname{ALT}(U / L)$ & 28 & & 22 \\
\hline Ferritin (ng/ml) & 7909 & & 4645 \\
\hline
\end{tabular}

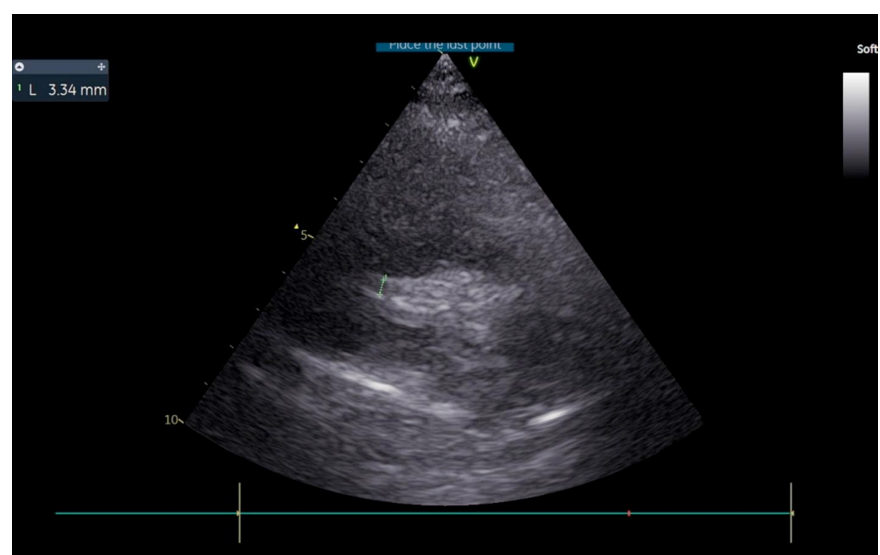

Figure 1: Initial echo image showing increased size of coronary arteries and mild pericardial effusion.

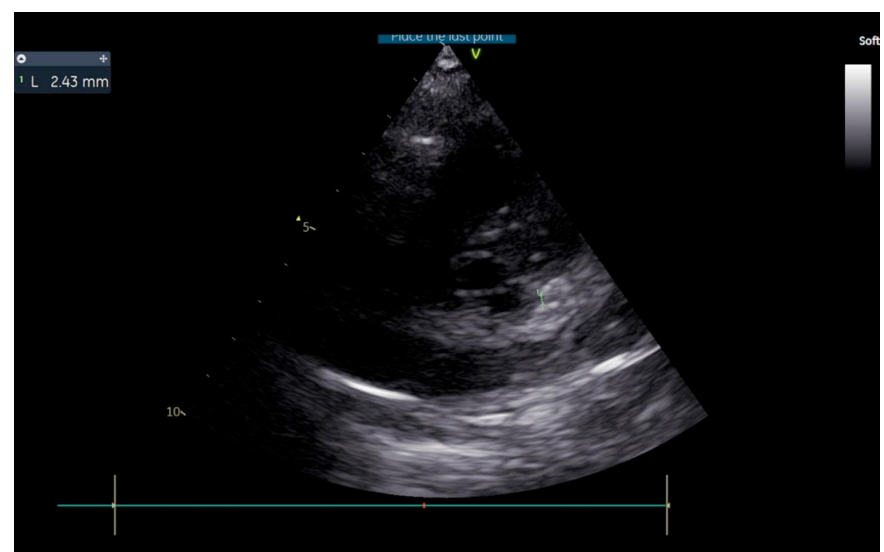

Figure 2: Follow-up echo image showing decreased size of coronary arteries.

The child was treated with intravenous ceftriaxone and supportive treatment of shock. IVIG, $2 \mathrm{~g}$ per $\mathrm{kg}$, was administered on the first day. High dose aspirin ( $80 \mathrm{mg} / \mathrm{kg} /$ day) was started. Due to recrudescence of fever after $48 \mathrm{hrs}$, methylprednisolone was given for three days. Symptoms improved after steroid administration. Echocardiogram was repeated on $5^{\text {th }}$ day and it showed improved pericardial effusion and static size of coronary arteries. Patient was discharged on low dose aspirin and called for follow-up. Follow-up scan after two weeks showed decreased size of coronaries arteries (Figure 2).

\section{DISCUSSION}

We present the case of a 7-year boy, who presented with fever and rash; initially diagnosis of KD was made, based on typical signs and symptoms of classical mucosal involvement and blanchable rash with non-purulent conjunctivitis and peripheral edema. ${ }^{2}$ IVIG was given on day 1 , but there was no improvement in clinical condition; and his inflammatory markers were high, which raised the suspicion of another possible etiology like MIS-C. ${ }^{1}$

Recently, eight cases of post-COVID inflammatory syndrome, also known as Kawasaki-like MIS-C, between the ages of 5-15 years, have been reported from different cities of Pakistan like Karachi, Lahore and Islamabad. The very first case outside Pakistan was reported on 26 April 2020, in the United Kingdom. ${ }^{4}$

CDC proposed a criteria for MIS-C diagnosis (age 0-19 years), which includes fever $\left(\geq 38.0^{\circ} \mathrm{C}\right.$ for $\geq 24$ hours), markedly elevated markers of inflammation (CRP, ESR, fibrinogen, pro-calcitonin (PCT), D-dimer, ferritin, LDH, interleukin 6 , raised neutrophils, low lymphocytes, and low albumin), multisystem organ involvement $>2$, no other alternate possible diagnosis and evidence of COVID-19 or recent exposure to a positive case. ${ }^{5}$

Several features of our patient raised the suspicion of MIS-C, though there was no history of exposure to COVID-19, like markedly elevated inflammatory markers, shock-like presentation requiring vasopressors, which is uncommon in KD, cardiac dysfunction, acute kidney injury (AKI) requiring diuretic infusion, stable respiratory status which itself a feature shared by patients with MIS-C, who often lack intrinsic respiratory disease. In our patient, COVID-PCR was negative but antibodies were positive, which was again consistent with MIS-C finding, representing delayed inflammatory response. ${ }^{3}$

Early management with high dose IVIG can avoid the majority of complications. ${ }^{5}$ Our patient did not respond on first dose of IVIG; hence, methylprednisolone was started after which the child dramatically improved. He was success- 
fully discharged after there was a downward trend of inflammatory markers and troponin I, no fever, no vasopressor or oxygen support till 48 hours.

On follow-up echo after two weeks, coronary artery dilation was decreased as compared to initial scan. There was no other complication reported.

It is advisable to have a high index of suspicion of KD; and timely intervention with blood tests, echocardiography and treatment within 10 days of disease can prevent cardiac complications.

The purpose of this case report is to stress upon the fact that KD should be kept in mind when evaluating a child with confirmed or suspected COVID-19, presenting with deteriorating symptoms as to pick it up early and avoid adverse outcomes.

\section{ACKNOWLEDGEMENT:}

The authors would like to thank the parents of the presented case for giving consent. We also thank our respected teachers Dr. Samina Shamim and Dr. Anwar-ul-Haq for their continuous guidance and support.

\section{CONFLICT OF INTEREST:}

The authors declared no conflict of interest.

\section{PATIENT'S CONSENT:}

Written and informed consent was obtained from the parents of the patient to publish this data.

\section{AUTHORS' CONTRIBUTION:}

SA: Selection of the case, conception of the case, collection of information and ensuring its accuracy and integrity, drafting the manuscript and final write-up.

FSA: Drafting of the manuscript and bibliography collection.

MA: Critical revision, final editing, overall supervision and final approval of the version to be published.

\section{REFERENCES}

1. Cherif MY, de Filette JMK, André S, Kamgang P, Richert B, Clevenbergh P. Coronavirus disease 2019- related Kawasaki-like disease in an adult: A case report. JAAD Case Reports 2020; 6(8): p. 780. doi: 10.1016/j.jdcr.2020.06.023.

2. Noorani M, Lakhani N. Kawasaki disease: Two case reports from the Aga Khan Hospital, Dar es Salaam-Tanzania. BMC Pediatrics 2018; 18(1):p.334. doi: 10.1186/s12887-0181306-5.

3. Loomba RS, Villarreal EG, Flores S. COVID-19 and hyperinflammatory syndrome in children: Kawasaki disease with macrophage activation syndrome in disguise? Cureus 2020; 12(8). doi: 10.7759/cureus.9515.

4. Khan KS, Ullah I. SARS-CoV-2 causes Kawasaki-like disease in children: Cases reported in Pakistan. J Medical Virol 2021; 93(1):20-1z. doi: 10.1002/jmv.26340.

5. Raut S, Roychowdhoury S, Bhakta S, Sarkar M, Nandi M. Incomplete Kawasaki disease as presentation of COVID-19 infection in an infant: A case report. J Tropical Pediatrics 2020; fmaa047. doi: 10.1093/tropej/fmaa047. 